\title{
Radiative and Electroweak Penguin B decays: Experimental Results
}

\section{John Walsh*}

Istituto Nazionale di Fisica Nucleare, Sezione di Pisa

E-mail: john.walsh@cern.ch

We present recent experimental results on radiative and electroweak penguin decays of $B$ mesons. In particular, searches for the rare decays $B^{0} \rightarrow \phi \gamma$ and $B^{+} \rightarrow K^{+} \tau^{+} \tau^{-}$and a study of CP violation in the channel $B^{0} \rightarrow K^{0} \pi^{+} \pi^{-} \gamma$ are presented. We also review results of angular analyses of the electroweak penguin decays $B^{0} \rightarrow K^{* 0} \mu^{+} \mu^{-}$and $B^{0} \rightarrow K^{* 0} e^{+} e^{-}$.

Flavor Physics and CP Violation,

6-9 June 2016

Caltech, Pasadena CA, USA

${ }^{*}$ Speaker. 


\section{Introduction}

Radiative and electroweak decays of $B$ mesons, in particular those involving the transitions $b \rightarrow s \gamma$ and $b \rightarrow s \ell^{+} \ell^{-}$, have proven to be powerful probes of New Physics (NP) in the flavour sector. These flavour-changing neutral current decays are prohibited at tree level in the SM, they only appear in loop diagrams. This makes them an excellent laboratory for studying the effects of
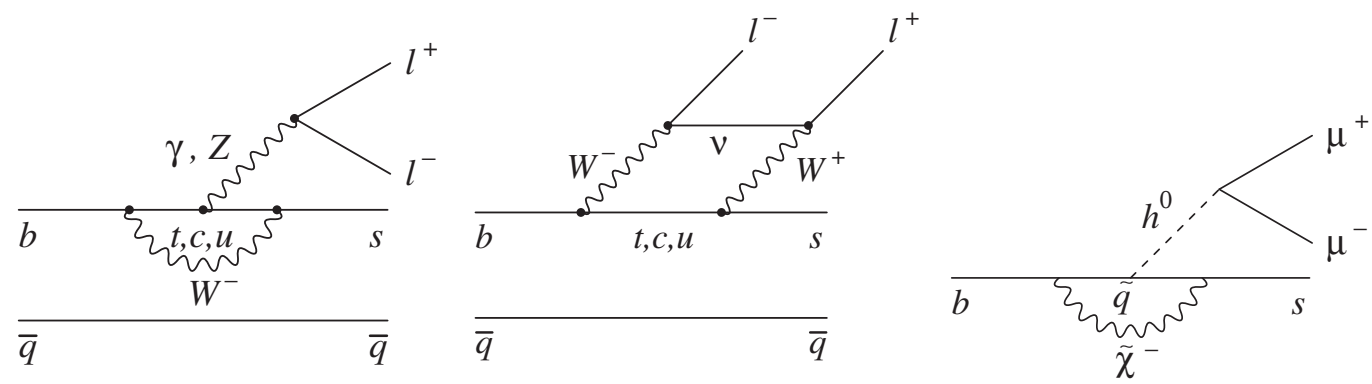

Figure 1: Lowest order SM diagrams $B \rightarrow X_{s} \ell^{+} \ell^{-}$decays (left and center) and a NP decay (right).

$\mathrm{NP}$, where new particles can enter the loops and have an appreciable effect on observable quantities. Figure 1 shows the lowest-order SM Feynman diagrams for the $b \rightarrow s \ell^{+} \ell^{-}$transition, along with a possible diagram in a New Physics scenario. A general review of radiative and electroweak penguin B physics can be found in section 17.9 of reference [1].

The following sections present recent experimental results on radiative and electroweak penguin $B$ decays.

\section{Search for CP violation in the decay $B^{0} \rightarrow K^{0} \pi^{+} \pi^{-} \gamma$}

In the Standard Model, the circular polarization of photons produced in $b \rightarrow s \gamma$ transitions is predominantly left-handed, with right-handed photons surpressed by a factor of $m_{s} / m_{b}$. This means that $B^{0}$ mesons (which contain a $\bar{b}$ anti-quark) decay mostly to right-handed photons, while $\bar{B}^{0}$ mesons produce mainly left-handed photons. One consequence of this is that mixing-induced $\mathrm{CP}$ violation in $B \rightarrow f_{C P} \gamma$ decays, where $f_{C P}$ is a CP eigenstate, is expected to be small. This situation is modified in NP models where photons of opposite helicity are produced [3].

The BaBar experiment has studied [2] the time-dependent CP asymmetry:

$$
A_{C P}(t)=\frac{\Gamma\left(\bar{B}^{0} \rightarrow K_{S}^{0} \rho^{0} \gamma\right)-\Gamma\left(B^{0} \rightarrow K_{S}^{0} \rho^{0} \gamma\right)}{\Gamma\left(\bar{B}^{0} \rightarrow K_{S}^{0} \rho^{0} \gamma\right)+\Gamma\left(B^{0} \rightarrow K_{S}^{0} \rho^{0} \gamma\right)}
$$

Neglecting detector effects, the asymmetry may be written as:

$$
A_{C P}(t)=S_{K_{S}^{0} \rho^{0} \gamma} \sin \left(\Delta m_{d} \Delta t\right)-C_{K_{S}^{0} \rho^{0} \gamma} \cos \left(\Delta m_{d} \Delta t\right),
$$

where $\Delta t$ is the decay time difference of the two $B$ mesons produced in the $\Upsilon(4 S) \rightarrow B^{0} \bar{B}^{0}$ decay and $S_{K_{S}^{0} \rho^{0} \gamma}$ and $C_{K_{S}^{0} \rho^{0} \gamma}$ are the CP-violation parameters to be measured.

One particular difficulty of the analysis is separating the CP eigenstate $B^{0} \rightarrow K_{S}^{0} \rho^{0} \gamma$ from other modes that contribute to the $B^{0} \rightarrow K_{S}^{0} \pi^{+} \pi^{-} \gamma$ final state. Due to the large natural width of the $\rho^{0}$, 
it is not possible to isolate the CP mode by selecting on the $\rho^{0}$ mass. A full Dalitz plot analysis of this channel is not feasible due to low statistics, so the solution adopted is to measure the effective CP-violating parameter $S_{K_{S}^{0} \pi^{+} \pi^{-} \gamma}$ and relate this quantity to $S_{K_{S}^{0} \rho^{0} \gamma}$ using a theoretical framework. The expression for this "dilution factor" is as follows [4]:

$$
\begin{aligned}
D_{K_{S}^{0} \rho \gamma} & \equiv \frac{S_{K_{S}^{0} \pi^{+} \pi^{-} \gamma}}{S_{K_{S}^{0} \rho^{0} \gamma}} \\
& =\frac{\int\left[\left|A_{\rho K_{S}^{0}}\right|^{2}-\left|A_{K^{*+} \pi^{-}}\right|^{2}-\left|A_{(K \pi)_{0}^{*+} \pi^{-}}\right|^{2}+2 \Re\left(A_{\rho K_{S}^{0}}^{*} A_{K^{*+} \pi^{-}}\right)+2 \Re\left(A_{\rho K_{S}^{0}}^{*} A_{(K \pi)_{0}^{*+} \pi^{-}}\right)\right] d m^{2}}{\int\left[\left|A_{\rho K_{S}^{0}}\right|^{2}+\left|A_{K^{*+} \pi^{-}}\right|^{2}+\left|A_{(K \pi)_{0}^{*+} \pi^{-}}\right|^{2}+2 \Re\left(A_{\rho K_{S}^{0}}^{*} A_{K^{*+} \pi^{-}}\right)+2 \Re\left(A_{\rho K_{S}^{0}}^{*} A_{(K \pi)_{0}^{*+} \pi^{-}}\right)\right] d m^{2}}
\end{aligned}
$$

Here $A_{R P}$ is the amplitude for the mode $R P$ where $R \in\left\{\rho^{0}, K^{* \pm},(K \pi)_{0}^{* \pm}\right\}$ is a hadronic resonance and $P$ is a pseudoscalar. The last element in the above list represents a possible $S$-wave contribution. These amplitudes are extracted from the isospin-related decay $B^{+} \rightarrow K^{+} \pi^{+} \pi^{-} \gamma$, due to the much larger statistics available for the charged channel. This assumes that the resonant amplitudes are the same in both modes.

The model employed for the amplitude analysis assumes the decay proceeds via $B^{+} \rightarrow K_{\text {res }}^{+} \gamma$, where $K_{\text {res }}$ is kaon resonance above the $K^{*}(892)$. Five different kaon resonances are included in the model and the contributions of each are determined from a fit to the $m_{K^{+} \pi^{+} \pi^{-}}$spectrum. Since $B\left(K_{\text {res }}^{+} \rightarrow K^{+} \pi^{-} \pi^{+}\right)$is not known for all the $K_{\text {res }}$ considered, the $m_{K \pi}$ spectrum is used to determine the $R P$ content of the $K^{+} \pi^{+} \pi^{-}$final state. Details of the employed models are provided in [2].

The $K^{+} \pi^{+} \pi^{-} \gamma$ invariant mass for selected events is shown in Fig. 2. A simultaneous fit to this distribution and the distributions of $\Delta E$ and a Fisher discriminant determine a yield of $2441 \pm 104$ signal events. The $m_{K \pi}$ distribution of the signal events, obtained using the Splots technique, is also shown. The results of the amplitude analysis allow the determination of the dilution factor
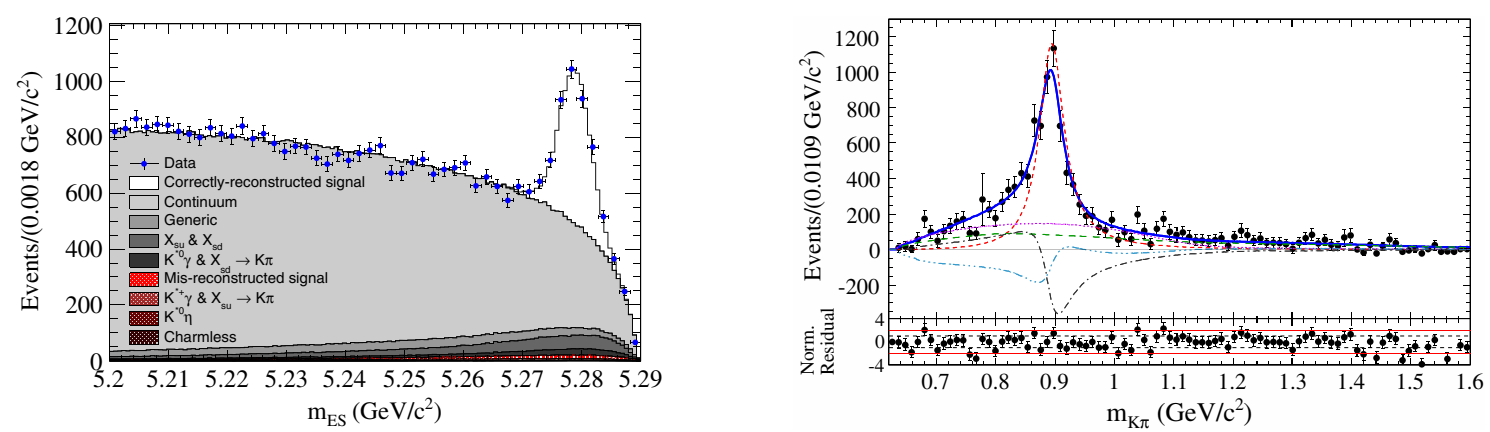

Figure 2: Left: mass distribution of $B^{+} \rightarrow K^{+} \pi^{+} \pi^{-} \gamma$ candidates. Right: distribution of $m_{K \pi}$ for signal events (obtained using the Splots technique). The blue solid curve corresponds to the total PDF fit projection. The small-dashed red, medium-dashed green and dotted magenta curves correspond to the $K^{* 0}, \rho^{0}$ and $S$ wave contributions, respectively. The dashed-dotted gray curve corresponds to the interference between the two P-wave components, and the dashed-triple-dotted light blue curve corresponds to the interference between the $\mathrm{S}$-wave and the $\rho^{0}$. 
(Eq. 2.3):

$$
D_{K_{S}^{0} \rho \gamma}=-0.78_{-0.17}^{+0.19}
$$

Next, the neutral channel $B^{0} \rightarrow K_{S}^{0} \pi^{+} \pi^{-} \gamma$ is analysed. A sample of $243 \pm 31$ signal events are identified. Flavour tagging is employed on the rest of the event to separate $B^{0}$ and $\bar{B}^{0}$ decays. The decay time difference $\Delta t$, which is determined by reconstructing the decay vertices of the two $B$ mesons, is added to the likelihood fit, along with the tagging information, and permits the extraction of the CP-violating paramters discussed above:

$$
\begin{aligned}
& S_{K_{S}^{0} \pi^{+} \pi^{-} \gamma}=0.14 \pm 0.25 \pm 0.03 \\
& C_{K_{S}^{0} \pi^{+} \pi^{-} \gamma}=-0.39 \pm 0.32 \pm 0.06
\end{aligned}
$$

Applying the dilution factor to the first of these yields the final result: $S_{K_{S}^{0} \rho^{0} \gamma}=-0.18 \pm 0.32 \pm 0.06$, which is in agreement with the Standard Model. This analysis is based on the full BaBar data set of $471 \times 10^{6} B \bar{B}$ pairs.

\section{The rare decay $B^{0} \rightarrow \phi \gamma$}

The Belle experiment has performed a search for the radiative decay $B^{0} \rightarrow \phi \gamma$ using their full data set corresponding to 772 million $B \bar{B}$ pairs [5]. This decay proceeds via the $b \rightarrow d \gamma$ transition and is therefore suppressed by a factor of about 25 (relative to $b \rightarrow s \gamma$ ) by CKM factors. The SM prediction for the branching fraction is $\sim 10^{-11}$, but large enhancements are expected in some physics models [6].

The event selection starts with a photon with energy greater than $2 \mathrm{GeV}$ in the $\Upsilon(4 S)$ (centerof-mass) system. Photons from the decays $\pi^{0} \rightarrow \gamma \gamma$ and $\eta \rightarrow \gamma \gamma$ are rejected by applying explicit vetoes. Pairs of charged kaons are used to reconstruct the $\phi$ meson, with $1.000<m_{K K}<1.039$ $\mathrm{GeV}$, which exploits the good mass resolution on the $\phi$ meson. Finally, a multi-variate selector reduces continuum background using event shape variables.

The signal yield is extracted using a simultaneous fit to four quantities: the beam-constrained $B$ mass, $\Delta E$, the output of the multi-variate selector and the helicity angle of the decay. The projections of the fits are shown in Fig. 3. No significant signal is found and a 90\% CL upper limit is obtained:

$$
B\left(B^{0} \rightarrow \phi \gamma\right)<1.0 \times 10^{-7} \quad 90 \% \mathrm{CL}
$$

This result improves on the previous best limit [7] by nearly an order of magnitude.

\section{The $B^{0} \rightarrow K^{* 0} \ell^{+} \ell^{-}$channels}

The $B^{0} \rightarrow K^{* 0} \ell^{+} \ell^{-}$channels have proven to be a powerful probe of physics beyond the standard model. From a phenomenological standpoint, they proceed via loops at lowest order, where New Physics can have large effects, as discussed in the Introduction. The richness of the final state allows the measurement of many observables that 1) can be well calculated within the SM and 2) can be significantly altered by the presence of NP. From the experimental viewpoint, these modes have generally clear signatures, with a lepton pair in the final state and relatively low backgrounds. 

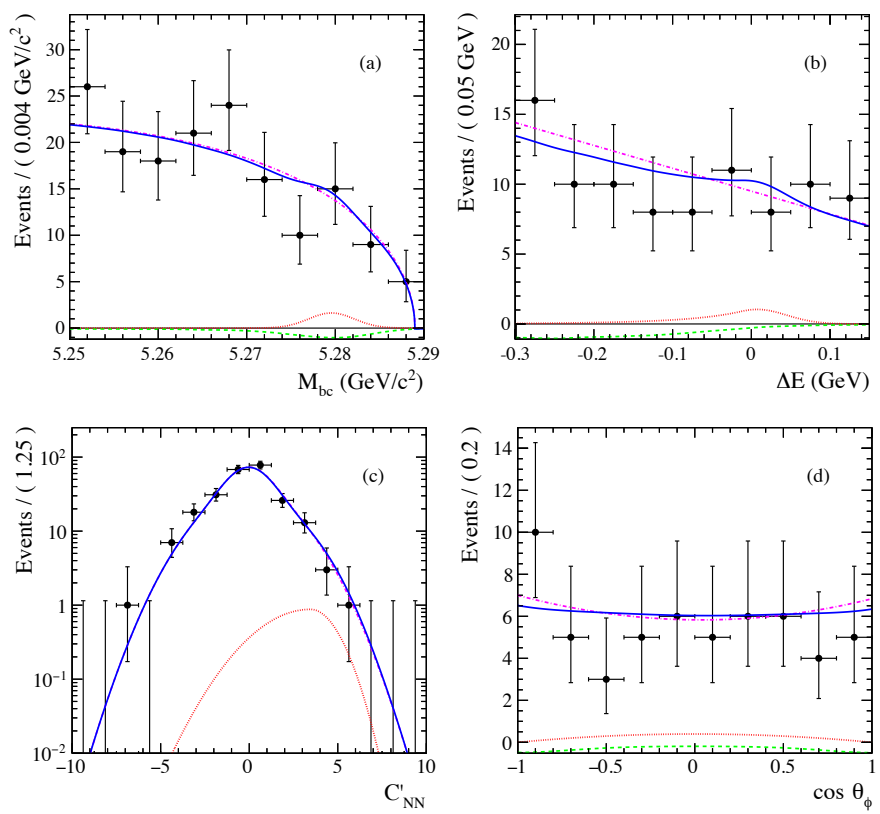

Figure 3: Projections of the 4-variable fit to the $B^{0} \rightarrow \phi \gamma$ candidates: a) the beam-constrainted mass, b) $\Delta E, \mathrm{c})$ neural net output and d) helicity angle. Selections are made on the variables not plotted to enhance the signal in any given projection. The blue curves represent the total fit, while the red curves represent the signal, the magenta curves the continuum events and the green curves the charmless background.

\subsection{Angular analysis of $B^{0} \rightarrow K^{* 0} \mu^{+} \mu^{-}$}

The advent of $\mathrm{LHCb}$ in recent years has produced great experimental advances in the $B^{0} \rightarrow$ $K^{* 0} \mu^{+} \mu^{-}$channel. We report here on an angular analysis of the $B^{0} \rightarrow K^{* 0} \mu^{+} \mu^{-}$channel based on the full Run 1 dataset, corresponding to an integrated luminosity of $3 \mathrm{fb}^{-1}$ [8].

The differential decay rate for the channel $B^{0} \rightarrow K^{* 0} \mu^{+} \mu^{-}$may be written:

$$
\begin{aligned}
\frac{d \Gamma}{d q^{2} d \Omega} \propto & \frac{3}{4}\left(1-F_{L}\right) \sin ^{2} \theta_{K}+F_{L} \cos ^{2} \theta_{K} \\
& +\frac{1}{4}\left(1-F_{L}\right) \sin ^{2} \theta_{K} \cos 2 \theta_{\ell} \\
& -F_{L} \cos ^{2} \theta_{K} \cos 2 \theta_{\ell}+S_{3} \sin ^{2} \theta_{K} \sin ^{2} \theta_{\ell} \cos 2 \phi \\
& +S_{4} \sin 2 \theta_{K} \sin 2 \theta_{\ell} \cos \phi+S_{5} \sin 2 \theta_{K} \sin \theta_{\ell} \cos \phi \\
& +\frac{4}{3} A_{F B} \sin ^{2} \theta_{K} \cos \theta_{\ell}+S_{7} \sin 2 \theta_{K} \sin \theta_{\ell} \sin \phi \\
& +S_{8} \sin 2 \theta_{K} \sin 2 \theta_{\ell} \sin \phi+S_{9} \sin ^{2} \theta_{K} \sin ^{2} \theta_{l} \sin 2 \phi
\end{aligned}
$$

where the three angles that define the final state topology are defined in Fig. 4. The quantities $F_{L}$ and $A_{F B}$ are the $K^{* 0}$ longitudinal polarization fraction and forward-backward lepton asymmetry, respectively, while the $S_{i}$ are related to the Wilson coefficients of the effective Hamiltonian and can be calculated in the SM. All of the quantities are extracted by performing a simultaneous fit to the distributions of the three decay angles. The fit also includes the $K^{+} \pi^{-} \mu^{+} \mu^{-}$invariant mass and the $K^{+} \pi^{-}$invariant mass. The fit is performed in seven bins of $q^{2} \equiv m_{\mu \mu}^{2}$, since theory predicts the 


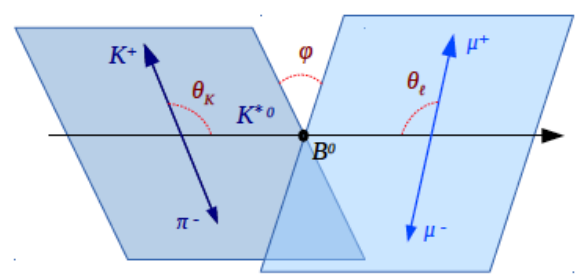

Figure 4: Definition of decay angles of $B^{0} \rightarrow K^{* 0} \mu^{+} \mu^{-}$decays.

variation of each of these coefficients with $q^{2}$. Indeed, the $q^{2}$ behavior of the coefficients provides important information in distinguishing NP models, should a deviation from the SM be found.

The possibility of an S-wave contribution to this decay, i.e., where the $K^{+} \pi^{-}$of the final state does not come from a $K^{* 0}$ decay, is included in the fit (Eq. 4.1 does not show the $\mathrm{S}$-wave contribution for simplicity).

Figure 5 shows the fit projections for the bin with $1.1<q^{2}<6.0 \mathrm{GeV}^{2}$.
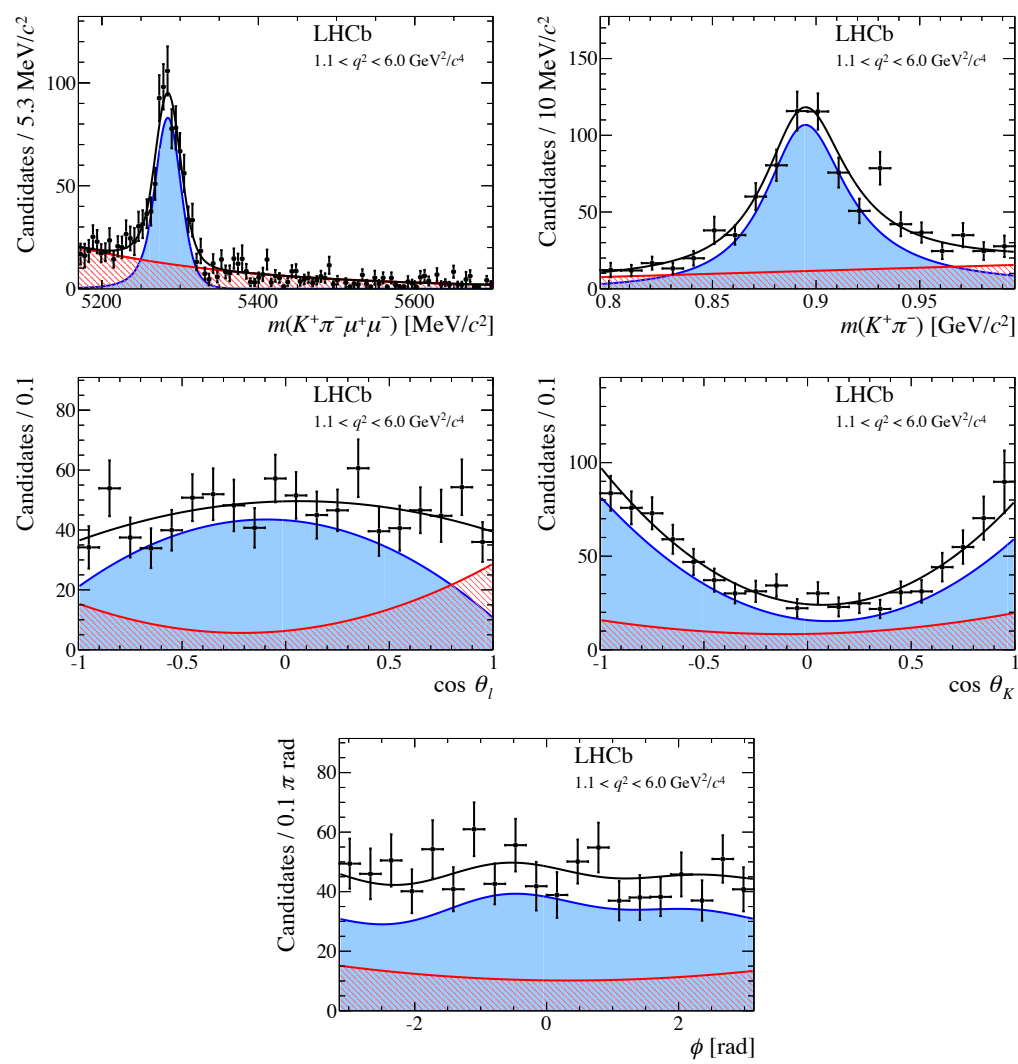

Figure 5: Projections of the 5D fit for the $q^{2}$ bin 1.1-6.0 $\mathrm{GeV}^{2}$. A mass cut of $\pm 50 \mathrm{MeV}$ around the known $B$ mass has been applied to the $m_{K \pi}$ and angular variable distributions. Candidates have been weighted to account for acceptance. The blue shaded area represents the signal, while the red hatched area denotes the background.

The results for $F_{L}$ and $A_{F B}$ as a function of $q^{2}$ are presented in Fig. 6. The experimental results 
are compared to the SM expections from ref. [9]. There is generally good agreement with the SM predictions. Additional variables, for which the theoretical uncertainties are signficiantly reduced,
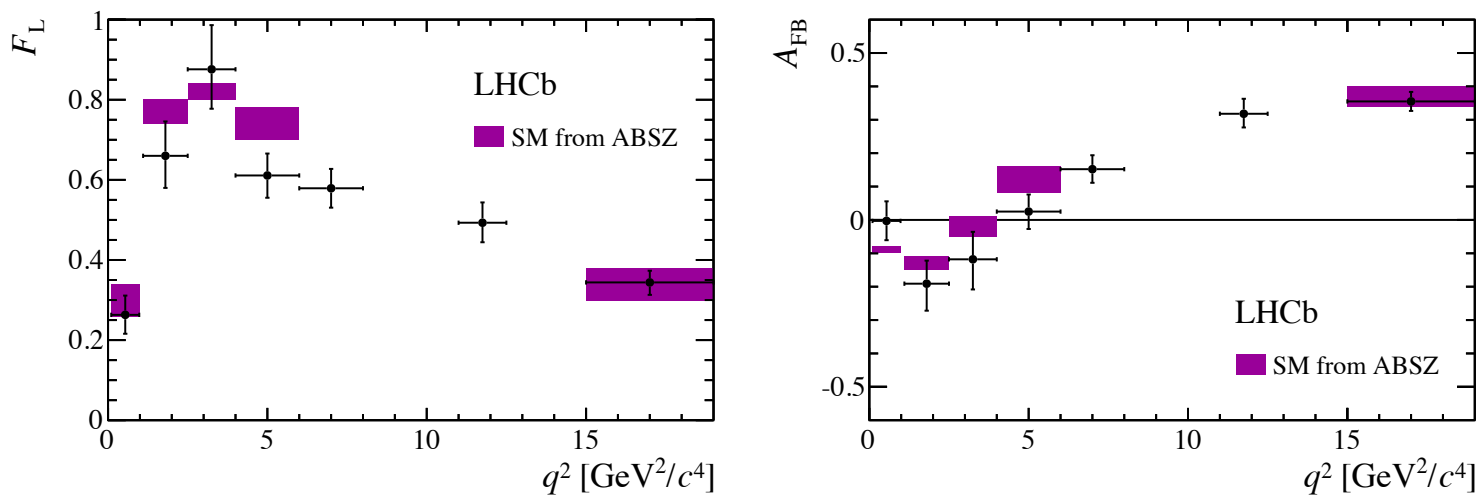

Figure 6: Results for $F_{L}$ (left) and $A_{F B}$ (right). The shaded areas represent the SM prediction from [9].

can be constructed from $F_{L}$ and the $S_{i}$. In Fig. 7 the results for $P_{5}^{\prime} \equiv S_{5} / \sqrt{F_{L}\left(1-F_{L}\right)}$ are shown, compared to the SM prediction [10]. Here a discrepancy with the SM calculation is observed in the fourth and fifth $q^{2}$ bins, with statistical significance of $2.8 \sigma$ and $3.0 \sigma$, respectively. Thus the discrepancy observed in the previous analysis [11], performed on one-third the data, is confirmed.

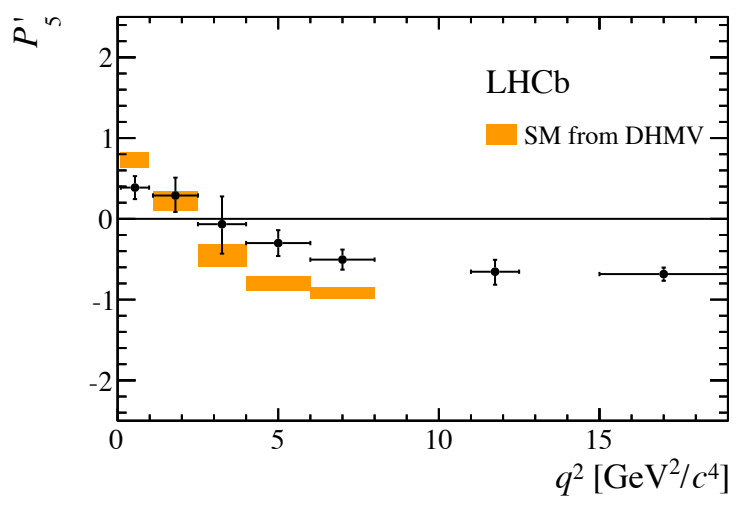

Figure 7: Results for optimised variable $P_{5}^{\prime}$. The shaded areas represent the SM prediction from [10].

For reasons of space, only a small subset of the results are presented here. The full results are presented in [8].

The CMS experiment has also performed an angular analysis of the $B^{0} \rightarrow K^{* 0} \mu^{+} \mu^{-}$channel [12]. They have lower statistics than LHCb and perform a somewhat simpler analysis, which requires fewer events. Figure 8 shows $F_{L}, A_{F B}$ and the branching fraction as a function of $q^{2}$. There is good agreement with the SM predictions.

This channel has also been studied at the B factory experiments Babar and Belle. They have significantly lower statistics, but can also perform the same analysis for the $B^{0} \rightarrow K^{* 0} e^{+} e^{-}$final state. Furthermore, because they can reconstruct neutral particles efficiently, this gives them access to the isospin-related channel $B^{+} \rightarrow K^{*+} \ell^{+} \ell^{-}$, which has either a $K_{S}^{0}$ or a $\pi^{0}$, arising from the $K^{*+}$ decay, in the final state. 

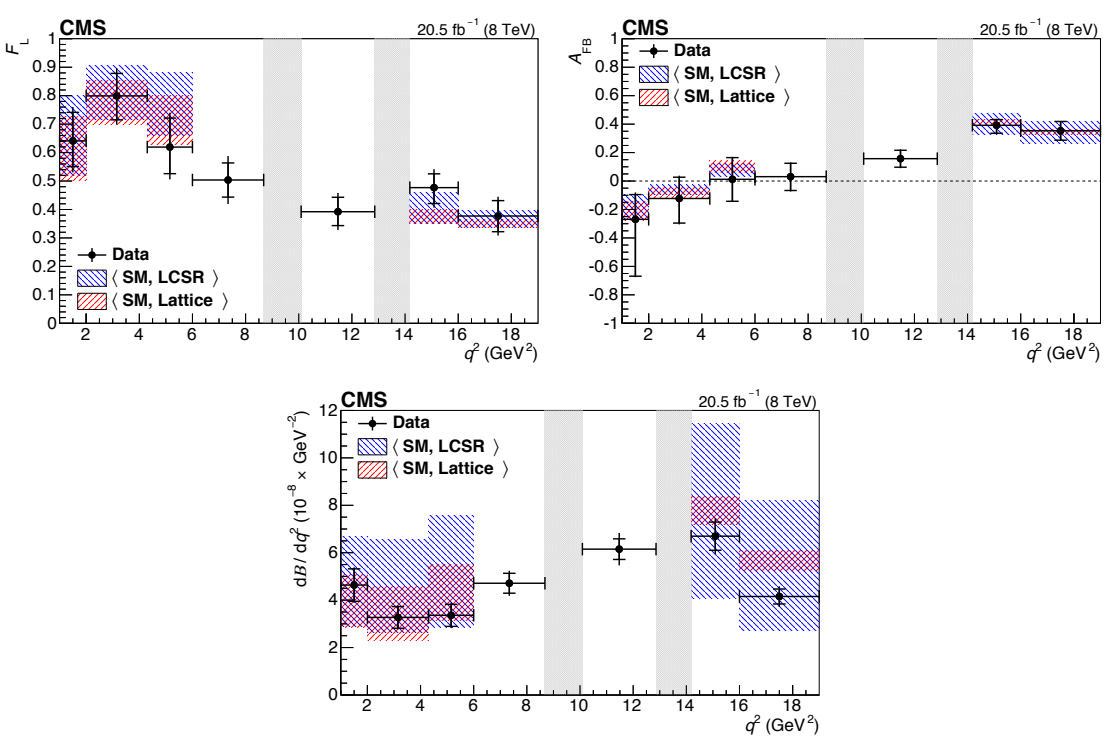

Figure 8: CMS results for $F_{L}$ (upper left), $A_{F B}$ (upper right) and the differential branching fraction as a function of $q^{2}$.

Figure 9 shows the Belle results for the optimized variable $P_{5}^{\prime}$ [13]. Interestingly, the Belle result favours the LHCb result for this observable and is also in disagreement with the SM. Babar

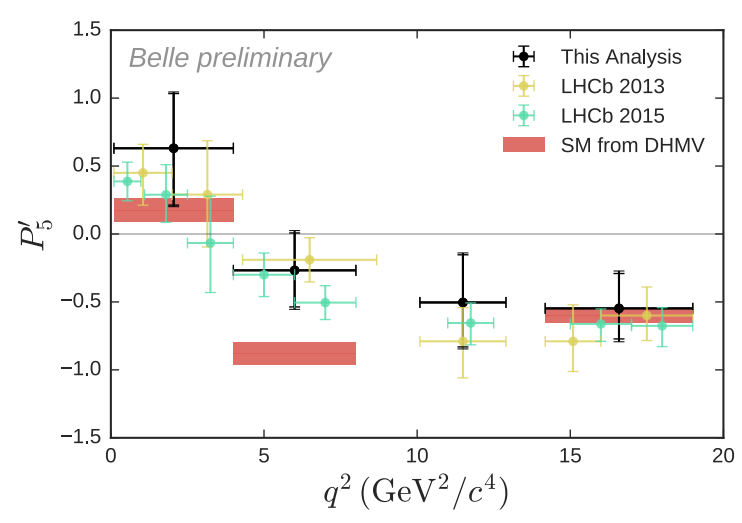

Figure 9: Belle results for $P_{5}^{\prime}$. The shaded areas represent the SM prediction from [10].

has measured $F_{L}$ and $A_{F B}$ separately for the $B^{0}$ and $B^{+}$modes [14]. Fig. 10 shows the results obtained for $F_{L}$ for $1<q^{2}<6 \mathrm{GeV}^{2}$, along with other experimental results and the SM prediction. There is some disagreement with the Standard Model and indeed a hint of a difference for the charged and neutral decays.

\subsection{Angular analysis of $B^{0} \rightarrow K^{* 0} e^{+} e^{-}$in the pole region}

LHCb has also performed an angular analysis of the channel $B^{0} \rightarrow K^{* 0} e^{+} e^{-}$, based on the full Run 1 data set, corresponding to $3 \mathrm{fb}^{-1}$ of integrated luminosity. In this case, only the lowest $q^{2}$ bin is examined, i.e., events with $0.002<q^{2}<1.120 \mathrm{GeV}^{2}$ [15]. This region is dominated by the 


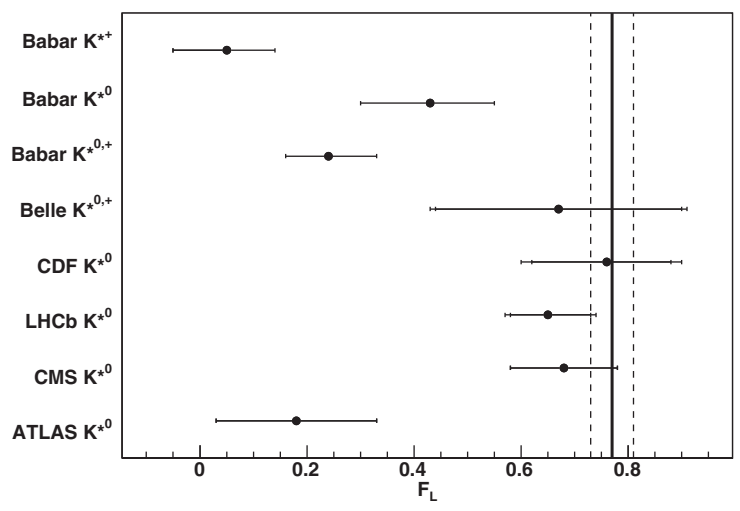

Figure 10: Results for $F_{L}$ in the range $1<q^{2}<6$ for the neutral and charged modes from Babar and compared with other experimental results. The vertical lines represent the SM prediction.

virtual photon contribution and thus allows searches for right-handed currents in NP scenarios. In terms of the Wilson coefficients of the effective Hamiltonian, the analysis is sensitive to $C_{7}$ and $C_{7}^{\prime}$.

As for the $B^{0} \rightarrow K^{* 0} \mu^{+} \mu^{-}$case, one can write down the differential decay rate as a function of the three angles in the decay:

$$
\begin{aligned}
\frac{d \Gamma}{d q^{2} d \Omega} \propto & \frac{3}{4}\left(1-F_{L}\right) \sin ^{2} \theta_{K}+F_{L} \cos ^{2} \theta_{K} \\
& +\left(\frac{1}{4}\left(1-F_{L}\right) \sin ^{2} \theta_{K}-F_{L} \cos ^{2} \theta_{K}\right) \cos 2 \theta_{\ell}+\frac{1}{2}\left(1-F_{L}\right) A_{T}^{(2)} \sin ^{2} \theta_{K} \sin ^{2} \theta_{\ell} \cos 2 \phi \\
& +\left(1-F_{L}\right) A_{T}^{R e} \sin ^{2} \theta_{K} \cos \theta_{\ell}+\frac{1}{2}\left(1-F_{L}\right) A_{T}^{I m} \sin ^{2} \theta_{K} \sin ^{2} \theta_{l} \sin 2 \phi,
\end{aligned}
$$

where the angles are defined as in Sec. 4.1, except that $\phi$ is "folded": $\phi \rightarrow \phi+\pi$ for $\phi<0$. There are four coefficients extracted by fitting the data: $F_{L}, A_{T}^{R e}=\frac{4}{3} A_{F B} /\left(1-F_{L}\right), A_{T}^{(2)}$ and $A_{T}^{I m}$. The latter two quantities are sensitive to the photon polarization and in the limit of very small $q^{2}$ are directly related to the Wilson coefficients $C_{7}$ and $C_{7}^{\prime}$ :

$$
A_{T}^{(2)}\left(q^{2} \rightarrow 0\right)=\frac{2 \operatorname{Re}\left(C_{7} C_{7}^{*}\right)}{\left|C_{7}\right|^{2}+\left|C_{7}^{\prime}\right|^{2}} \quad A_{T}^{I m}\left(q^{2} \rightarrow 0\right)=\frac{2 \operatorname{Im}\left(C_{7} C_{7}^{*}\right)}{\left|C_{7}\right|^{2}+\left|C_{7}^{\prime}\right|^{2}}
$$

Experimentally, this channel is more challenging than the $B^{0} \rightarrow K^{* 0} \mu^{+} \mu^{-}$channel discussed earlier, for two primary reasons: 1) the excellent LHCb muon trigger is not applicaable for this channel and 2) the significant bremsstrahlung of the final state electrons degrades the mass resolution of the recontructed $B$ meson. A significant background comes from photon conversions to very low-mass $e^{+} e^{-}$pairs. These are suppressed by applying a minimum cut on the di-electron mass. An estimated $4 \%$ of the selected signal consists of this background.

Figure 11 shows the distribution of the invariant $B$ mass and the three angular distributions for the selected $B^{0} \rightarrow K^{* 0} e^{+} e^{-}$events. The results obtained from the fit are: 

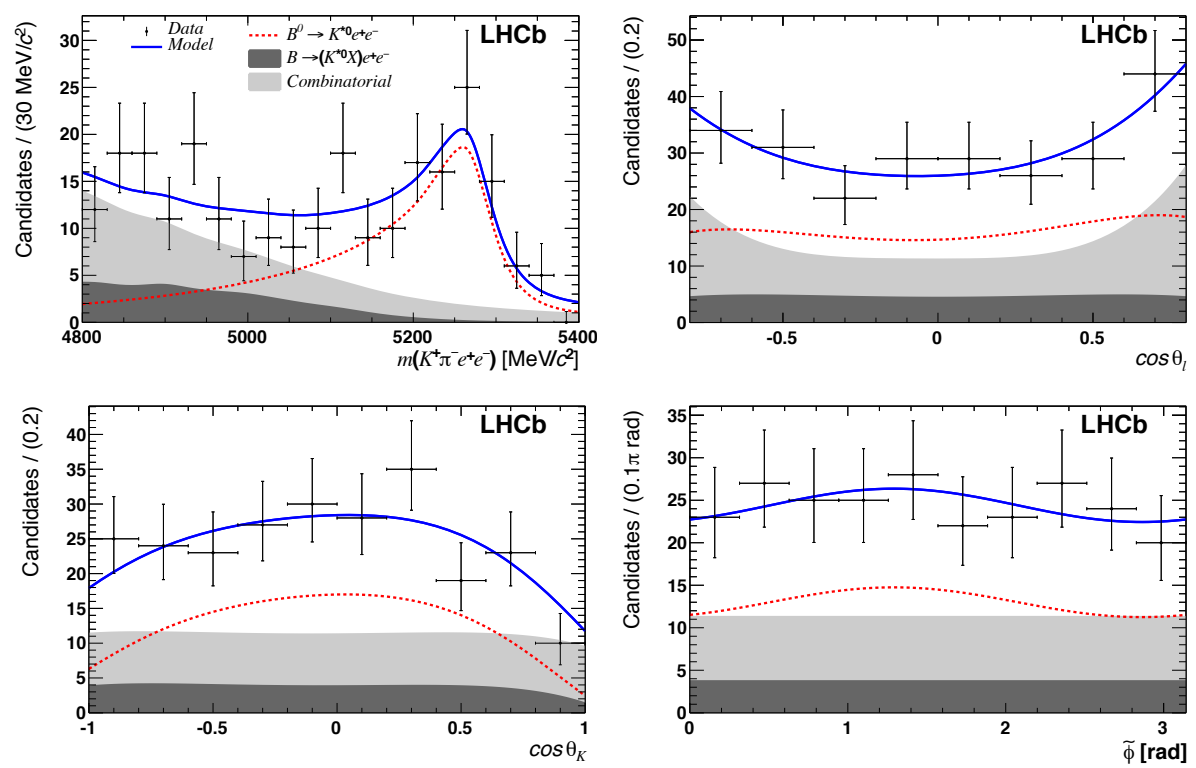

Figure 11: Projections of 4D fit to the $B$ candidate invariant mass and the three decay angles. The widening of the mass distribution relative to the $B^{0} \rightarrow K^{* 0} \mu^{+} \mu^{-}$case due to bremsstrahlung is evident.

$$
\begin{aligned}
F_{L} & =0.16 \pm 0.06 \pm 0.03 \\
A_{T}^{(2)} & =-0.23 \pm 0.23 \pm 0.05 \\
A_{T}^{I m} & =0.14 \pm 0.22 \pm 0.05 \\
A_{T}^{R e} & =0.10 \pm 0.18 \pm 0.05
\end{aligned}
$$

These are the first measurements of these quantities in this $q^{2}$ range. They are compatible with Standard Model expectations and in particular with $C_{7}^{\prime}=0$.

\section{Search for $B^{+} \rightarrow K^{+} \tau^{+} \tau^{-}$}

Babar has performed a search for the decay $B^{+} \rightarrow K^{+} \tau^{+} \tau^{-}$, which is related to the wellstudied decays with a pair of muons or electrons in the final state [16]. The $\tau$ channel is interesting because of the third lepton's large mass: there is a potential NP contribution to the decay via a neutral Higgs boson that couples more strongly to higher mass leptons. Furthermore, given recent results on $R_{K}=B(B \rightarrow K \mu \mu) / B(B \rightarrow K e e)$ [17], which exhibit a tension with the SM expectation, there is heightened interest to measure the rate to $B \rightarrow K \tau \tau$.

This mode is very challenging experimentally: there are multiple neutrinos in the final state and very few kinematic handles on the signal decay. At the $e^{+} e^{-} \mathrm{B}$ factories the technique of tagging the other $B$ makes it possible to recontruct this decay. B-tagging involves fully reconstructing the "other" B in the event. For this analysis, hadronic final states were considered for the tag B, starting with either a charmed or charmonium meson and then adding up to five additional pions and kaons. Once the tag B has been reconstructed, its daughters are removed from consideration and the signal decay is looked for among the remaining tracks in the event. Leptonic $\tau$ decays are considered, so the final state signature consists of a charged kaon, a pair of leptons (either $\mu$ or $e$ ) 
and a significant amount of missing energy due to the lost neutrinos. Explicit vetoes are employed to remove backgrounds from $J / \psi, D^{0}$ and $\pi^{0}$ decays.

The primary remaining background comes from semileptonic cascade decays: $B \rightarrow D \ell v$ followed by $D \rightarrow K \ell v$. This background, which has the same final state particles as the signal decay, is reduced using a multi-layer preceptron (MLP) selector with seven input variables. Figure 12 shows the distribution of the MLP output for data and estimates of the background obtained from simulation. The shape of a possible signal is also shown (red histogram, arbitrary scale). The MLP

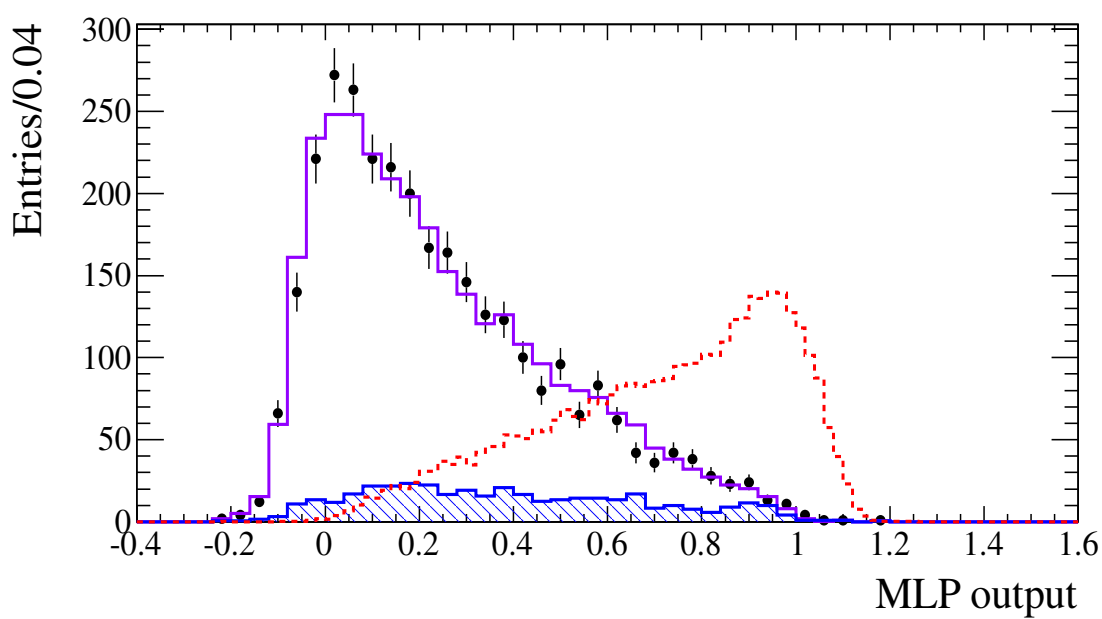

Figure 12: Distribution of the MLP output for data (points), combinatorial background (blue histogram) and peaking background (purple histogram). The red histogram shows the shape of the signal on an arbitrary scale.

cut was placed at 0.7 for the $e e$ and $\mu \mu$ candidates and at 0.75 for the $e \mu$ candidates. The results are presented in Table 1. For the $e e$ and $\mu \mu$ modes the number of observed events is in good agreement with the background estimates. However, an excess is seen in the $e \mu$ channel, with respect to the expected background, with a statistical significance of 3.7 $\sigma$. Inspection of the events in question shows no indication of signal-like behavior, nor evident problems with the background modeling. Taken together the three channels are in agreement with background expectations within $2 \sigma$. Thus, an upper limit is derived:

$$
B\left(B^{+} \rightarrow K^{+} \tau^{+} \tau^{-}\right)<2.25 \times 10^{-3} \quad 90 \% \mathrm{CL}
$$

This analysis is based on the full BaBar data set of 471 million $B \bar{B}$ pairs.

\section{Global fits to $b \rightarrow s \ell \ell$ and $b \rightarrow s \gamma$}

The wealth of results in radiative and electroweak penguin decays has given rise to several phenomenological efforts to extract information on NP from the group of measurements $[18,19]$. The current set of results favors a New Physics contribution to the Wilson coefficient $C_{9}$. This is depicted in Fig. 13, which shows the fitted results for $C_{9}^{\mathrm{NP}}$ vs. $C_{9}^{\prime \mathrm{NP}}$ and $C_{9}^{\mathrm{NP}}$ vs. $C_{10}^{\mathrm{NP}}$. The super-script "NP" indicates that the SM contribution has been subtracted out such that in the SM all $C_{i}^{\mathrm{NP}}=0$. 


\begin{tabular}{lccc}
\hline \hline & $e^{+} e^{-}$ & $\mu^{+} \mu^{-}$ & $e^{ \pm} \mu^{\mp}$ \\
\hline \hline$N_{\text {bkg }}^{i}$ & $49.4 \pm 2.4 \pm 2.9$ & $45.8 \pm 2.4 \pm 3.2$ & $59.2 \pm 2.8 \pm 3.5$ \\
$\varepsilon_{\text {sig }}^{i}\left(\times 10^{-5}\right)$ & $1.1 \pm 0.2 \pm 0.1$ & $1.3 \pm 0.2 \pm 0.1$ & $2.1 \pm 0.2 \pm 0.2$ \\
$N_{\text {obs }}^{i}$ & 45 & 39 & 92 \\
Significance $(\sigma)$ & -0.6 & -0.9 & 3.7 \\
\hline \hline
\end{tabular}

Table 1: Expected background yields, $N_{\text {bkg }}^{i}$, signal efficiencies, $\varepsilon_{\text {sig }}^{i}$, number of observed data events, $N_{\text {obs }}^{i}$, and signed significance for each signal mode. Quoted uncertainties are statistical and systematic.
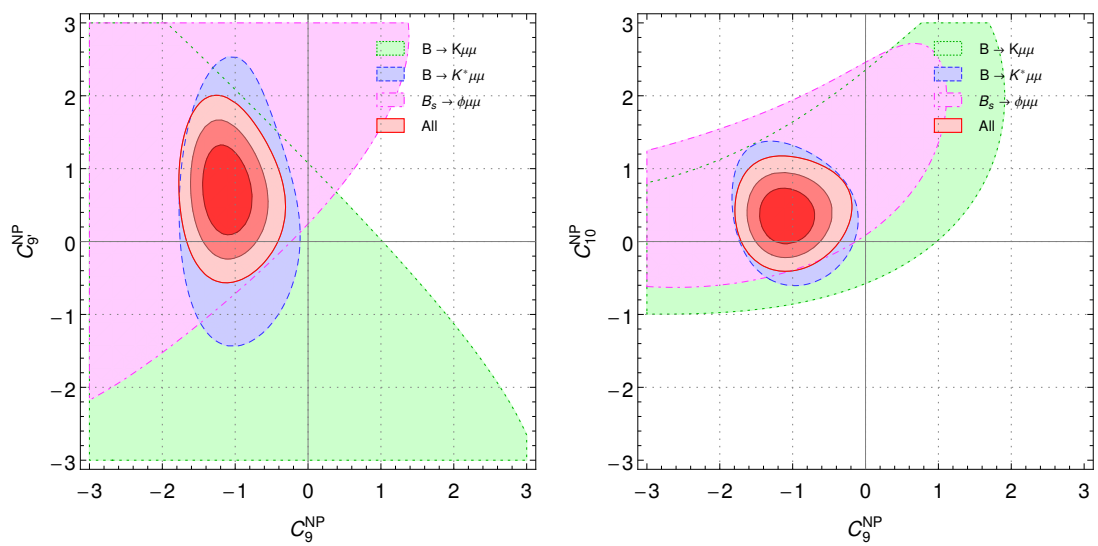

Figure 13: Results of global fits to $b \rightarrow s \gamma$ and $b \rightarrow s \ell \ell$ measurements. Taken from ref. [18]

Additional details on the global fits were presented at this conference by Sébastien DescotesGenon [20].

\section{Conclusions}

There is a wealth of information on radiative penguin decays of $B$ mesons coming from both the LHC and the B factories. We have presented searches for right-handed currents via photon polarization as well as comprehensive angular analyses of the $B \rightarrow K^{(*)} \ell^{+} \ell^{-}$channels. These results put powerful contraints on the NP models and currently show some tension with the Standard Model in the $P_{5}^{\prime}$ observable.

$\mathrm{LHCb}$ is busy accumulating data in Run 2 and expects to more than double the Run 1 data set by 2018. The B factory experiments are producing their final papers after having stopped data taking some years ago. However, the next generation of B factories is not far off: Belle II expects to commence taking data for physics in 2018.

\section{Acknowledgments}

I would like to express my gratitude to my colleagues Professors Dave Hitlin and Frank Porter, and their staff, for organizing an excellent and enjoyable conference. 


\section{References}

[1] Ed. A.J. Bevan, B. Golob, Th. Mannel, S. Prell, and B.D. Yabsley, The Physics of the B Factories, Eur. Phys. J. C74 (2014) 3026, [arXiv:1406.6311]

[2] P. del Amo Sanchez et al. (BaBar Collaboration), Time-dependent analysis of $B^{0} \rightarrow K_{S}^{0} \pi^{-} \pi^{+} \gamma$ decays and studies of the $K^{+} \pi^{-} \pi^{+}$system in $B^{+} \rightarrow K^{+} \pi^{-} \pi^{+} \gamma$ decays, Phys. Rev. D93, 052013 (2016) [arXiv:1512.03579].

[3] K. Fujikawa and A. Yamada, Test of the chiral structure of the top-bottom charged current by the process $b \rightarrow s \gamma$, Phys. Rev. D 49 (1994) 5890;

K.S. Babu, K. Fujikawa and A. Yamada, Constraints on left-right symmetric models from the process $b \rightarrow s \gamma$, Phys. Lett. B 333 (1994) 196;

P. Cho and M. Misiak, $b \rightarrow s \gamma$ decay in $S U(2)_{L} \times S U(2)_{R} \times U(1)$ extensions of the standard model, Phys. Rev. D 49 (1994) 5894.

[4] J. Hebinger, E. Kou and F.-S. Yu, Note on the dilution factor for the time-dependent CP asymmetry measurement in $B^{0} \rightarrow \rho^{0} K_{S} \gamma$ decays, report LAL-15-75 (2015).

[5] Z. King et al. (Belle Collaboration), Search for the decay $B^{0} \rightarrow \phi \gamma$, Phys. Rev D93 111101 (2016), [arXiv:1603.06546].

[6] X.Q. Li, G.R. Lu, R.M. Wang and Y. Yang, The rare $\bar{B}_{d}^{0} \rightarrow \phi \gamma$ decays in Standard Model and as a probe of R-parity violation, Eur. Phys. J. C 36 (2004) 97;

J. Hua, C. Kim and Y. Li, Annihilation-Type Charmless Radiative Decays of B Meson in Non-universal Z' Model, Eur. Phys. J. C 69 (2010) 139.

[7] B. Aubert et al. (BaBar Collaboration), Search for the radiative decay $B^{0} \rightarrow \phi \gamma$, Phys. Rev. D72, 091103 (2005) [arXiv: hep/ex-0501.1038].

[8] R. Aaij et al. (LHCb Collaboration), Angular analysis of the $B^{0} \rightarrow K^{* 0} \mu^{+} \mu^{-}$decay using $3 f^{-} 1$ of integrated luminosity, JHEP 02 (2016) 104, [arXiv: 1512.0444 ].

[9] W. Altmannshofer and D.M. Straub, New physics in $b \rightarrow$ s transitions after LHC Run 1, Eur. Phys. J C74 (2014) 2927, [arXiv:1401.2145].

[10] S. Descotes-Genon, L. Hofer, J. Matias and J. Virto, On the impact of power corrections in the prediction of $B \rightarrow K^{*} \mu^{+} \mu^{-}$observables, JHEP 12 (2014) 125, [arXiv: 1407 . 8526].

[11] R. Aaij et al. (LHCb Collaboration), Measurement of form-factor-independent observables in the decay $B^{0} \rightarrow K^{* 0} \mu^{+} \mu^{-}$, Phys. Rev. Lett. 111 (2013) 191801, [arXiv:1308.1707].

[12] V. Khachatryan et al. (CMS Collaboration), Angular analysis of the decay $B^{0} \rightarrow K^{* 0} \mu^{+} \mu^{-}$from pp collisions at $\sqrt{s}=8 \mathrm{TeV}$, Phys. Lett. B753 (2016) 424, [arXiv: 1507 .08126].

[13] A. Abdesselam et al. (Belle Collaboration), Angular Analysis of $B^{0} \rightarrow K^{*}(892)^{0} \ell^{+} \ell^{-}$, [arXiv: 1604.04042$]$.

[14] J.P. Lees et al. (BaBar Collaboration), Measurement of Angular Asymmetries in the Decays $B \rightarrow K^{*} \ell^{+} \ell^{-}$, Phys. Rev. D93, (2016) 52015, [arXiv: 1508 .07960].

[15] R. Aaij, et al. (LHCb Collaboration), Angular analysis of the $B^{0} \rightarrow K^{* 0} e^{+} e^{-}$decay in the low- $q^{2}$ region, JHEP 04 (2015) 064, [arXiv: 1501 .03038].

[16] J.P. Lees et al. (BaBar Collaboration), Search for $B^{+} \rightarrow K^{+} \tau^{+} \tau^{-}$at the BaBar experiment, [arXiv:1605.09637]. 
[17] R. Aaij, et al. (LHCb Collaboration), Test of lepton universality using $B^{+} \rightarrow K^{+} \ell^{+} \ell^{-}$decays, Phys. Rev. Lett. 13 (2014) 151601, [arXiv: 1406 . 6482].

[18] S. Descotes-Genon, L. Hofer, J. Matias and J. Virto, Global analysis of $b \rightarrow$ sll anomalies, JHEP 06 (2016) 092, [arXiv:1510.04239].

[19] W. Altmannshofer and D.M. Straub, Implications of $b \rightarrow$ s measurements, [arXiv: 1503.06199]; M. Ciuchini et al., $B \rightarrow K^{*} \ell^{+} \ell^{-}$decays at large recoil in the Standard Model: a theoretical reappraisal, [arXiv:1512.07157];

T. Hurth, F. Mahmoudi and S. Neshatpour, On the anomalies in the lastest LHCb data, Nucl. Phys. B 909 (2016) 737, [arXiv: 1603.00865$]$.

[20] S. Descotes-Genon, proceedings from this conference. 